\title{
Hemangioendotelioma epitelioide hepático primario: un simulador de metástasis
}

\author{
F. J. Torres Gómez, F. Rivera Hueto, F. J. Torres Olivera
}

\section{Resumen}

El hemangioendotelioma epitelioide hepático es un tumor raro en el adulto, de etiología desconocida y origen vascular, que afecta más a mujeres. Suelen cursar asintomáticos o con síntomas poco específicos y con frecuencia su diagnóstico es casual. La lesión suele ser múltiple, afecta a ambos lóbulos hepáticos y su tamaño es muy variable. Las características de la lesión en las técnicas diagnósticas por imagen son superponibles a las de un proceso metastático. El diagnóstico histológico requiere estudio inmunohistoquímico complementario y no aporta criterios de valor pronóstico. La evolución es imprevisible y letal en el 50\% de los pacientes. La extirpación quirúrgica de la lesión y el transplante hepático son las opciones terapéuticas más útiles.

Describimos las características clínico-patológicas de esta rara lesión, aportando un caso diagnosticado inicialmente de metástasis hepática.

\section{Palabras clave:}

Hemangioendotelioma epitelioide. Hígado. Transplante hepático.

Oncología, 2005; 28 (6):288-292 


\section{Summary}

Hepatic epithelioid hemangioendothelioma is a rare vascular tumor of adults of unknown etiology, that is more common in women. It is usually asymptomatic or presents only non specific symptoms, making the diagnosis frequently casual. The lesions are usually multifocal and of variable sizes, involving both lobes. The characteristics of the lesions shown by the imaging diagnostic techniques are superposable to those of of the metastatic disease. The histologic diagnosis requires immunohistological studies and does not contribute with prognostic criteria. The course is unexpected and $50 \%$ of the patients die of the disease. Surgery and hepatic transplant are the most useful therapeutic options. We describe the clinicopathologic characteristics of this rare lesion and contribute with a case, thay was initially diagnosed as hepatic metastatic disease.

Key words: Epithelioid hemangioendothelioma. Liver. Transplant.

\section{Introducción}

El hemangioendotelioma epitelioide es una neoplasia poco frecuente, cuya localización hepática es excepcional en el adulto ${ }^{1-5}$. La lesión fue inicialmente descrita en el pulmón como tumor bronquioloalveolar intravascular y considerada histogenéticamente como epitelial ${ }^{6}$. Posteriormente se demostró su naturaleza endotelial, ultraestructuralmente ${ }^{7}, \mathrm{y}$ por métodos inmunohistoquímicos ${ }^{8}$. En 1982, Weiss y Enzinger ${ }^{9}$ proponen el término hemangioendotelioma epitelioide, que ha prevalecido hasta la actualidad y señalan su pronóstico incierto. Su etiologia es desconocida 4 .

Aportamos las características clínico-patológicas de un caso de hemangioendotelioma epitelioide hepático primario, confirmado con estudio inmunohistoquímico y diagnosticado clínica y radiológicamente de metástasis hepática, enfatizando la importancia del diagnóstico anatomopatológico en el adecuado manejo terapéutico de estos pacientes.

\section{Presentación del caso}

Mujer de 56 años que consulta por malestar abdominal y astenia de varios meses de evolución. La exploración física no revela hallazgos patológicos. El estudio analítico (hematológico y bioquímico) no presenta alteraciones. La radiología simple de abdomen revela pequeñas imágenes radiopacas de densidad cálcica en el área hepática, que se corresponden en la ecografía y la TAC con varias lesiones hipoe- coicas e hipodensas en ambos lóbulos hepáticos, con un tamaño comprendido entre 0,5 y $1,5 \mathrm{~cm}$ de diámetro, que fueron interpretadas como metástasis. Las exploraciones para descartar un tumor primario mamario y gastrointestinal fueron negativas. La paciente se somete a una laparotomia exploradora en la que se visualizan varias lesiones blanquecinas que protuyen en la superficie de ambos lóbulos hepáti$\cos$, de superficie lisa, de las que se toman muestras para estudio anatomopatológico.

\section{Estudio anatomopatológico}

Las muestras corresponden a dos fragmentos cuneiformes de 1,5 y $1 \mathrm{~cm}$ de dimensiones máximas, de color blanquecino en la zona central y marrón en la periferia. Al corte se aprecian dos lesiones blanquecinas, de límites definidos y consistencia firme, rodeadas de tejido de color marrón. Las muestras de tejido seleccionadas para estudio microscópico fueron fijadas en formol acuoso al 10\% durante 24 horas e incluidas en parafina. Se obtuvieron cortes histológicos de 3 micras de espesor que se tiñeron con hematoxilina-eosina. Otros cortes se montaron sobre portas cargados eléctricamente y se destinaron a estudio inmunohistoquímico, que se realizó de forma automatizada en un aparato tipo ventana.

Microscópicamente se advierte una lesión neoplásica con patrón de crecimiento sólido y márgenes infiltrativos que ocupa parcial o totalmente las luces venulares y sinusoidales (Fig. 1 A y 1 B), respetando los espacios porta y en cierta medida las trabécu- 


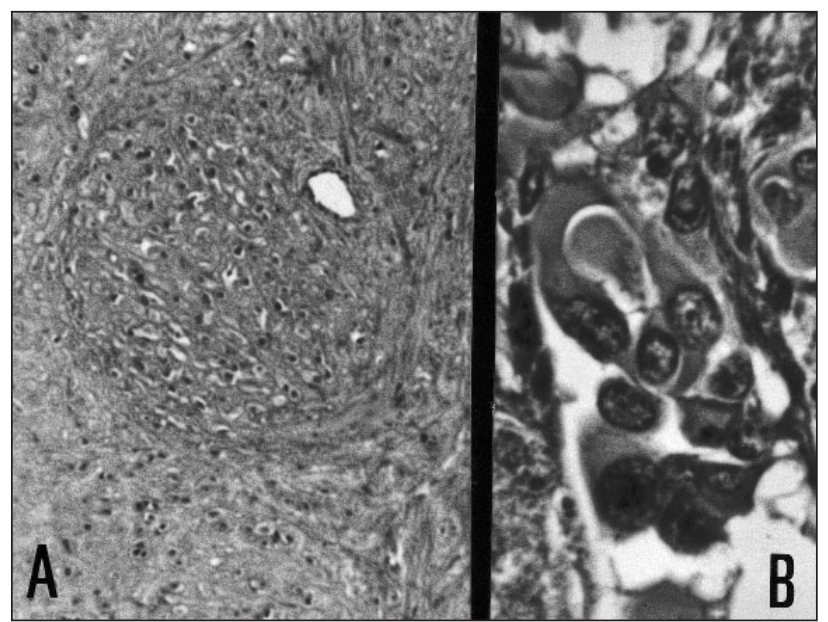

Figura 1. Hemangioendotelioma hepático: A) Oclusión tumoral de vénula. H.E. 100x. B) Sustitución de luces sinusoidales por las células tumorales. H.E. 400x.

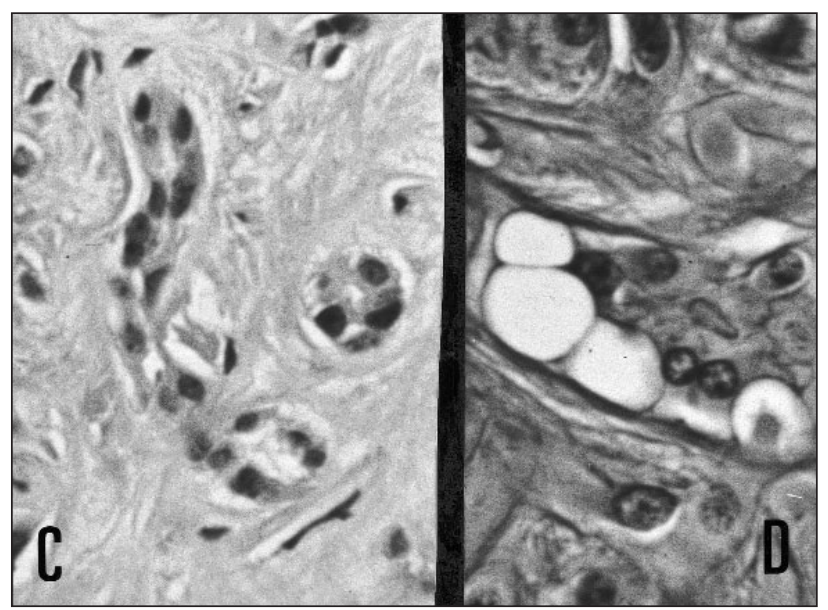

Figura 2. Hemangioendotelioma hepático: C) Area tumoral de predominio fibroso con preservación de conductillos. D) Célula tumoral multivacuolada. H.E. 400x.

las hepáticas y conductillos biliares. Alternan áreas celulares con otras de aspecto fibroso (Fig. 2 C). Las células neoplásicas dominantes exhiben citoplasmas amplios y densos, de bordes bien definidos, a veces con vacuolas únicas o múltiples (Fig. 2 D), ocasionalmente ocupadas por hematíes y núcleos vesiculares con nucleolos evidentes y aisladas mitosis. La mayoría de las células tumorales expresan inmunopositividad fuerte frente a los marcadores vasculares FVIII, CD 31 y CD 34 y ocasionalmente frente al anticuerpo citoqueratina.

La paciente no ha recibido tratamiento y las lesiones han permanecido estables hasta el momento actual, año y medio después del diagnóstico.

\section{Discusión}

El hemangioendotelioma epitelioide hepático es un tumor infrecuente ${ }^{2,4,5}$ que afecta dos veces más a mujeres de edad media que a varones ${ }^{4}$ y su manifestación clínica es inexistente o poco llamativa en la mayoría de los casos ${ }^{1,2,4}$. Los datos analíticos normales de nuestra paciente discrepan de la frecuente elevación de la fosfatasa alcalina sérica (69\%), de la alaninaminotransferasa $(37 \%)$ y de la aspartatoaminotransferasa (48\%) observada en estos pacientes ${ }^{2,4}$. Aunque las calcificaciones hepáticas son observadas en el 23\% de los casos, las técnicas de imagen aportan pocas características diferenciales ${ }^{10,4}$, por lo que raramente permiten un diagnóstico inicial ${ }^{11,12}$. Por ello, a pesar de ciertas limitaciones, el método más fiable de diagnóstico es el estudio anatomopatológi$\mathrm{CO}^{2,4}$. El mejor conocimiento de las características morfológicas de estas lesiones en el material obtenido por PAAF ${ }^{13-15}$, añade un procedimiento diagnóstico muy útil.

Las características histopatológicas del hemangioendotelioma epitelioide hepático son indistinguibles de las que esta lesión presenta en otras localizaciones más usuales (tejidos blandos y pulmón) ${ }^{9}$ y el $10 \%$ de las lesiones pulmonares metastatizan en hígado, por lo que la exclusión de un tumor primario en estas localizaciones es obligada. El diagnóstico histológico con técnicas de rutina es difícil y sólo el $25 \%$ de los casos, correcto ${ }^{4}$. El diagnóstico diferencial se plantea con lesiones neoplásicas y no neoplásicas. Las neoplasias malignas más frecuentemente confundidas con el hemangioendotelioma epitelioide hepático son los tumores epiteliales esclerosantes del hígado (colangiocarcinoma y hepatocarcinoma), las metástasis de carcinomas, especialmente los de célula en "anillo de sello" y el angiosarcoma². Incluso con estudio inmunohistoquímico se pueden plantear problemas de diagnóstico diferencial ya que a veces las células tumorales expresan marcadores epiteliales $^{4}$. El angiosarcoma presenta mayor definición histológica, es raro en mujeres en esta localización ${ }^{16}$ y tiene un comportamiento muy agresivo. La similitud terminológica puede inducir a confundir el hemangioendotelioma epitelioide hepático con el hemangioendotelioma infantil, una entidad que afecta casi exclusivamente a niños, con características clinicopatológicas distintivas ${ }^{2}, 17$. El hemangioendotelioma epitelioide hepático puede simular la enfer- 
medad venoclusiva ${ }^{2,18}$, debido a la invasión tumoral de las vénulas terminales hepáticas y presentarse clínicamente como un síndrome de Budd-Chiari ${ }^{19-21}$.

No existen criterios anatomopatológicos con valor pronóstico, ni macroscópicos ni histológicos, y la evolución es impredecible ${ }^{4,22}$.

La capacidad de recurrencias locales $(1,5 \%)$, y de metástasis (27\%) y la corta supervivencia en el $43 \%$ de los casos, definen el hemangioendotelioma epitelioide hepático como una neoplasia maligna. En el $87 \%$ de los casos letales, el fallecimiento fue atribuido al tumor y el $63 \%$ presentaban metástasis ${ }^{4}$. La supervivencia es muy variable y se han descrito regresiones espontáneas de las lesiones, dependiente, posiblemente, de la extensa oclusión vascular por el tumor ${ }^{4,23}$.

La peculiar historia natural del hemangioendotelioma epitelioide hepático dificulta la valoración de la efectividad de los tratamientos. El 50\% de los pacientes sin tratamiento sobreviven más de 5 años, y la existencia de metástasis no influye en el alargamiento o acortamiento del período de superviven$\mathrm{cia}^{4}$. En pacientes con lesiones únicas o escasas, la extirpación quirúrgica es el tratamiento de elección ${ }^{3}$. Los pacientes con lesiones múltiples o extensas, que son la mayoría, deben transplantarse, sin que la existencia de metástasis sea una contraindicación ${ }^{24,25}$. La quimioterapia y radioterapia no han influido significativamente en el curso de la enfermedad ${ }^{3,4}$. La embolización arterial, muy útil en el tratamiento del hepatocarcinoma, no ha podido ser evaluada aún ${ }^{26}$. En pacientes asintomáticos no se aconseja tratamiento ${ }^{6}$.

Nuestra paciente sobrevive sin tratamiento 18 meses después del diagnóstico inicial.

Correspondencia:

Dr. F. J. Torres Gómez

C/ Matahacas, 18A - 1B

E-41003 Sevilla

e-mail: javiertorresgomez@yahoo.es

\section{Bibliografía}

1. Ditese O, Davies S, Williams R et al. Malignant epithelioid hemangioendothelioma of the liver: a clinicopathological and histochemical study of 12 cases. Histopatolopy 1989; 15: $225-37$

2. Ishak K, Sesterhenn I, Goodman Z et al. Epithelioid hemangioendothelioma of the liver. A clinicopathologic and follow-up study of 32 cases. Hum Pathol 1984; 15:83952.

3. Laufer J, Zimmermann A, Krehenbuhl L et al. Epithelioid hemangioendothelioma of the liver a rare hepatic tumor. Cancer 1996; 78:2318-27.

4. Makhlouf H, Ishak K, Goodman Z. Epithelioid hemangioendothelioma of the liver: a clinicopathologic study of 137 cases. Cancer 1999; 85:562-82.

5. Pokharna R, Garg P, Gupta S et al. Primary epithelioid hemangioendothelioma of the liver: case report and rewiew of the literature. J Clin Pathol 1997; 50:1029-31.

6. Dall D, Liebow A. Intravascular bronchioloalveolar tumor. Am J Pathol 1975; 78:6a-7a.

7. Corrin B, Manners B, Millard M et al. Histogenesis of the so-called "intravascular bronchioloalveolar tumor". J Pathol 1979; 128:163-7.

8. Weldon Linne V, Victor T, Christ M et al. Angiogenic nature of "the intravascular bronchioloalveolar tumor" of de lung: an electron microscopic study. Arch Pathol Lab Med 1986; 165:174-9.

9. Weiss S, Enzinger F. Epithelioid hemangioendothelioma: a vascular tumor often mistaken for carcinoma. Cancer 1982; 50: $970-81$.

10. Fulcher A, Sterling R. Hepatic neoplasms: computed tomography and magnetic resonance features. J Clin Gastroenterol 2002; 34:463-71.

11. Radin D, Craig I, Coletti P. et al. Hepatic epithelioid hemangioendothelioma. Radiology 1988,169,145 - 148.

12. Furui S, Itei Y, Ohmoto K et al. Hepatic epithelioid hemangioendothelioma: a report of five cases. Radiology 1989: 171:63-8.

13. Cho N, Lee K, Jeong M. Cytologic evaluation of primary malignant vascular tumors of the liver. One case each of angiosarcoma and epitheliod hemangioendothelioma. Acta Cytol 1997; 41:1468-76.

14. Gambacorta M, Bonacina E. Epithelioid hemangioendothelioma: report of a case dignosed by fine-needie aspiration. Diagn Cytopathol 1989; 5:207-10.

15. Sosiow R, Yin P, Steinberg C et al. Cytopathologic features of hepatic epitelioid hemangioendothelioma. Diagn Cytophatol 1997; 17:50-63.

16. Locker G, Doroshow J, Zwelling L et al. The clinical features of hepatic angiosarcoma: a report of four cases and review of the English literature. Medicine 1979; 58:4864.

17. Stocker J. An approach to handling pediatric liver tomors. Am J Clin Pathol 1998; 109:867-72. 
18. Eckstein R, Ravich R. Epithelioid hemangioendothelioma of the liver: report of two cases histologically mimicking veno-oclusive disease. Pathology 1986; 18:45962.

19. Fukayama M, Nihei Z, Takizawa T et al. Malignant epithelioid hemangioendothelioma of the liver, spreading through the hepatic veins. Virchows Arch A Pathol Anat Histopathol 1984; 404:275-87.

20. Hayashi Y, Inagaki K, Hirota S et al. Epithelioid hemangioendothelioma with marked liver deformity and secondary Budd-Chiari syndrome pathological and radiological correlation. Pathol Int 1999; 49:547-52.

21. Waish M, Hytiroglou P, Thug S et al. Epithelioid hemangioendothelioma of the liver mimicking Budd-Chiari syndrome. Arch Pathol Lab Med 1998; 122:846-48.

22. Urrutia A, Rego M, Ojanguren I et al. Epithelioid heman- gioendothelioma: a neoplasm with irregular behaviour. Med Clin 2000; 115:357.

23. Anthony P. Tumors and tumor-like lesions of the liver an biliary tract. In: MacSween R, Anthony P, Scheuer P editors. Pathology of the liver. 2nd edition. Edinburgh. Churchill Livingstone 1987; 574-645.

24. Marino I, Todo S, Tzakis A et al. Treatment of hepatic epithelioid hemangioendothelioma with lover transplantation. Cancer 1988; 62:2079-84.

25. Kelleher M, Iwatsuki S, Sheahan D. Epithelioid hemangioendothelioma of the liver: clinicopathological correlation of 10 cases treated by orthotopic liver transplantation. Am J Surg Pathol 1989; 13:999-1008.

26. Kawabe T, Tagawa K, Unuma $\mathrm{T}$ et al. Hepatic epithelioid hemangioendothelioma in a young female. Diag Dis Sci 1987; 32:1422-7. 